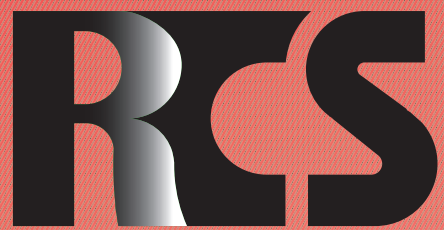

Depósito legal ppi $201502 Z U 4662$

Esta publicación científica en formato digital es continuidad de la revista impresa Depósito Legal: pp $197402 Z U 789$

- ISSN: 1315-9518 • ISSN-E: 2477-9431

Revista de Ciencias Sociales

Universidad del Zulia. Revista de la Facultad de Ciencias Económicas y Sociales Vol. XXVII. No. 2

Abril-Junio 2021

Esta publicación científica en formato digital es continuidad de la revista impresa Depósito Legal: pp $197402 Z$ Z789 ISSN: 1315-9518 


\title{
Proceso administrativo y sostenibilidad empresarial del sector hotelero de la parroquia Crucita, Manabí-Ecuador
}

\author{
Márquez Ortiz, Luis Enrique* \\ Viteri Mero, Morelia Jessenia** \\ Useche Castro, Lelly María*** \\ Cuétara Sánchez, Leonardo Manuel $^{* * * *}$
}

\section{Resumen}

La industria hotelera en la provincia de Manabí (Ecuador) toma auge a principios de los noventa con la disminución del valor comercial del camarón, destacándose por encima de otras actividades económicas en la provincia. Ahora, la industria hotelera de Manabí tiene la necesidad de un proceso administrativo encaminado hacia escenarios positivos y competitivos; ambientalmente sustentables. Por tanto, el objetivo de esta investigación fue determinar la relación entre el proceso administrativo y la sostenibilidad empresarial en el sector hotelero de la parroquia Crucita en Manabí-Ecuador. Para ello, se implementó una metodología estructurada en etapas, la cual se aplicó a un grupo de siete hoteles en la parroquia Crucita. Utilizando la investigación documental, el método Delphi y el coeficiente de correlación de Pearson, se logró una lista de indicadores para medir cada variable de estudio; así como el nivel de aplicación en los hoteles estudiados; encontrando que la mayoría de ellos no aplican el proceso administrativo de forma global e integral, no consideran la sostenibilidad como estrategia de la organización, además, los hoteles con mejor rendimiento sostenible son los más competitivos a nivel general; concluyendo en una relación directamente proporcional entre la sostenibilidad empresarial y el nivel de aplicación del proceso administrativo.

Palabras clave: Proceso administrativo; sostenibilidad empresarial; sector hotelero; competitividad; desarrollo sostenible.

Doctor en Ciencias Económicas. Profesor Principal Tiempo Completo de la Universidad Técnica de Manabí, Ecuador. E-mail: lemoortiz@yahoo.es (iD) ORCID: https://orcid.org/0000-0002-4801-1337

** Estudiante de Postgrado de la Universidad Internacional de la Rioja, España. Ingeniero Comercial. E-mail: moreliaviteri@gmail.com iD ORCID: https://orcid.org/0000-0002-5750-191X

*** Doctora en Estadística. Docente de Estadística y Demografía de la Universidad Técnica de Manabí, Ecuador. E-mail: lelly.estadistica@utm.edu.ec iD ORCID: https://orcid.org/0000-0002-4294-9009

***** Doctor en Ciencias Económicas. Profesor Contratado Tiempo Completo de la Universidad Técnica de Manabí, Ecuador. E-mail: leonardocuetara@gmail.com iD ORCID: https://orcid.org/0000-0001-9623-1193 


\title{
Administrative process and business sustainability of the hotel sector af the Crucita parish, Manabí-Ecuador
}

\begin{abstract}
The hotel industry in the province of Manabí (Ecuador) boomed in the early nineties with the decrease in the commercial value of shrimp, standing out above other economic activities in the province. Now, the Manabí hotel industry needs an administrative process aimed at positive and competitive scenarios; environmentally sustainable. Therefore, the objective of this research was to determine the relationship between the administrative process and business sustainability in the hotel sector of the Crucita parish in Manabí-Ecuador. For this, a methodology structured in stages was implemented, which was applied to a group of seven hotels in the Crucita parish. Using documentary research, the Delphi method and Pearson's correlation coefficient, a list of indicators was obtained to measure each study variable; as well as the level of application in the hotels studied; Finding that most of them do not apply the administrative process in a global and integral way, they do not consider sustainability as a strategy of the organization, in addition, the hotels with the best sustainable performance are the most competitive at a general level; concluding in a directly proportional relationship between business sustainability and the level of application of the administrative process.
\end{abstract}

Keywords: Administrative process; business sustainability; hotel sector; competitiveness; sustainable development.

\section{Introducción}

El turismo en Ecuador, se ha convertido en los últimos años en una alternativa para alcanzar el crecimiento económico del país, con un aporte de 2.392 millones de dólares, lo que representa un crecimiento del $27,8 \%$ con relación al 2018 (Organización Mundial del Turismo, 2019). El aporte directo del turismo al Producto Interno Bruto ecuatoriano (PIB) fue del $2,3 \%$, pero si se añaden actividades relacionadas, el aporte total sube al 5,6\%, en este caso, 6.070 millones de dólares (Ministerio de Turismo del Ecuador [MINTUR], 2019).

En Ecuador, el turismo se mantiene como la tercera fuente de ingresos no petroleros, después del banano y el camarón, de acuerdo con estimaciones realizadas a partir de los datos de Balanza de pagos del Banco Central del Ecuador (Ministerio de Turismo del Ecuador, 2019). Con relación a la generación de empleo, la ministra de turismo del Ecuador,
Rossi Prado de Holguín, explicó: Mientras que en el año 2018 se generaron 480.000 empleos directos y 112.000 indirectos, en el 2019 esta cifra aumentó a 512.000 trabajos directos, y 189.000 indirectos (La Hora, 2019).

Para la provincia de Manabí, el turismo es una de las actividades más productivas, está relacionado directamente con la generación de empleo, el desarrollo de la región y la captación de divisa. En el 2016, el nivel de consumo turístico total en la Provincia fue de 132,6 millones de dólares. Conforme a los resultados definitivos proporcionados por la Coordinación de Estadísticas e Investigación del Ministerio de Turismo (MINTUR), en el 2017, Manabí es la tercera provincia con mayores llegadas de turistas a nivel nacional, representando un $15 \%$ del total (MINTUR, 2020). Como se demuestra, el turismo para Manabí se ha convertido en una actividad estratégica para lograr disminuir los niveles de pobreza en la Provincia y conservar los recursos naturales y 
culturales que hacen posible la rentabilidad así como competitividad de la industria turística Manabita.

Con relación al sector hotelero del Ecuador, Serrano (2018), destaca que el mismo creció entre el 2016 y 2017 en un $28 \%$, generando 374,2 millones de dólares, con nuevas inversiones y un escenario positivo para la industria hotelera ecuatoriana. De los datos que se poseen hasta el 3er trimestre del 2017, existían 498.385 personas empleadas dentro de la actividad de alojamiento y restauración, lo que equivale a un crecimiento del $2,4 \%$ con relación al año 2016 , esta cifra representa el $6,4 \%$ del total de empleados en la economía nacional (Alcívar, 2018). Estos datos demuestran la importancia de la industria hotelera como sector estratégico para generar empleo, desarrollo y captación de divisas para la economía nacional.

Según Vélez y Poveda (2017), la empresa hotelera asentada en Manabí, figura desde los años 1980, la cual toma auge a partir del año 1990 con la pérdida de valor de la producción de camarón, y de esta manera la actividad turística comienza a posicionarse sobre las demás actividades económicas en la Provincia. En el 2015, Manabí contaba con 649 establecimientos de alojamiento, superada solamente por la provincia de Pichincha, con 850 establecimientos (Corporación Financiera Nacional, 2017).

En este sentido, se comprende, que la industria hotelera en Manabí necesita de procesos administrativos que la conduzcan a escenarios positivos y competitivos. La pérdida en términos de calidad y competitividad de los productos y servicios, se han vuelto factores preocupantes para las organizaciones. Cada estrategia para el diseño y desarrollo de los productos y servicios de la industria hotelera manabita es puesta en marcha mediante la acción empresarial, que, para ser eficaz, como lo expresa Chiavenato (2001), necesariamente debe ser planeada, organizada, dirigida y controlada.

Cabe destacar, que el sector hotelero es parte del sistema turístico, lo que indica que las empresas hoteleras deben considerar la sostenibilidad como estrategia de la organización, y utilizar el proceso administrativo para lograr este objetivo. Como se manifiesta en el "PLANDETUR 2020" de Ecuador, en el objetivo estratégico No. 4:

Es necesario generar una oferta turística sostenible y competitiva potenciando los recursos humanos, naturales y culturales, junto con la innovación tecnológica aplicada a los componentes de infraestructuras, equipamientos, facilidades y servicios, para garantizar una experiencia turística integral de los visitantes nacionales e internacionales. (Banco Interamericano de Desarrollo [BID], 2007, p.128)

La sostenibilidad para las empresas, es un enfoque de negocio orientado a generar valor a largo plazo para los accionistas y los demás grupos de interés, a través de una gestión equilibrada en términos económicos, sociales y ambientales (Gutiérrez, 2009). Visto de esta forma, para el sector hotelero de la provincia de Manabí, la sostenibilidad empresarial debe incluir elementos de responsabilidad social, producción y consumo sostenible, monitoreo constante de los impactos empresariales en los aspectos sociales y ambientales, así como acciones responsables de los stakeholders hacia la comunidad que los acoge. Si bien es cierto, Epstein (2009), explica que: "los administradores han reconocido la importancia de los inputs y el compromiso de los stakeholders, así como el impacto potencial en la rentabilidad de las empresas a largo plazo" (p.24).

El sector hotelero de Crucita, no es una actividad que funcione en forma independiente del proceso de desarrollo nacional, también debe contribuir en el contexto del desarrollo sostenible del Ecuador. Los empresarios hoteleros, deben considerar la mejor manera de ofrecer un servicio, apoyándose en el proceso administrativo para indicar la planificación, organización, dirección y control de la organización, en función de hacer del sector hotelero una actividad económica, social y ambientalmente sostenible. La gestión del proceso administrativo se da donde quiera que exista una organización, y el éxito de una empresa se debe a la importancia que le otorgue 
a este proceso dentro de la administración, como lo afirmaba Henry Fayol, fundador de la teoría clásica, al decir que: "el proceso administrativo es una función básica de la empresa" (Chiavenato, 2006. p.134).

En esta investigación, se implementa una metodología para definir un conjunto de indicadores relacionados al proceso administrativo: Planificación, organización, dirección y control. Igualmente, se proponen indicadores para medir la variable sostenibilidad empresarial, y a partir del coeficiente de correlación de Pearson se conoce la relación que existe entre las variables seleccionadas. El trabajo tiene importantes implicaciones económicas, sociales y ambientales para la sociedad, puesto que, está orientado a determinar la relación entre el proceso administrativo y la sostenibilidad empresarial del sector hotelero de la parroquia Crucita, cantón Portoviejo.

El estudio permite conocer el nivel de aplicación del proceso administrativo, como herramienta para definir y colocar en práctica la estrategia de sostenibilidad empresarial del sector hotelero de la parroquia Crucita. Como lo expresa el "PLANDETUR 2020", en su objetivo estratégico No. 4 (BID, 2007), y el Objetivo 12, de los Objetivos de Desarrollo Sostenible (ODS), este último declara que:

Se trata de hacer más y mejor con menos, aumentar las ganancias netas de bienestar de las actividades económicas mediante la reducción del uso de recursos, la degradación y la contaminación a lo largo de todo el ciclo de vida, al tiempo que se aumenta la calidad de vida. (Organización de las Naciones Unidas [ONU], 2020. p.2).

\section{El proceso administrativo}

Posiblemente, los primeros aspectos teóricos sobre el significado de proceso administrativo son expuestos por Frederick Taylor, en los principios de la administración científica, a inicios del Siglo XX. Para Taylor (1911), la gerencia debe respetar cuatro principios, estos son: Principio de planeación, de preparación, de control y principio de ejecución. Años más tarde, el francés Henry Fayol, en su teoría clásica de la administración, identifica las funciones de la administración, a saber: Prever, organizar, dirigir, coordinar y controlar; explicaba que las funciones de la administración eran las más importantes, puesto que éstas integran y coordinan las demás funciones de la organización (Fayol, 1987).

En la actualidad, estos principios y funciones se han fusionado para formar lo que en teoría se define como proceso administrativo, y según Chiavenato (2006), "es el nombre que se le adjudica al conjunto de funciones de la administración, e incluye: planear, organizar, dirigir y controlar (p.131). Igualmente, los autores Hernández y Palafox (2012), definen el proceso administrativo como "el instrumento teórico básico que le permite al administrador profesional comprender la dinámica del funcionamiento de una empresa (organización). Sirve para diseñarla, conceptualizarla, manejarla, mejorarla" (p.165).

La importancia del proceso administrativo para las organizaciones ha sido destacada por diferentes teorías, desde la teoría de la administración científica, hace más de cien años, hasta los nuevos enfoques administrativos surgidos a partir de 1990, estos últimos representados por la teoría de la complejidad, innovación, benchmarking, la administración del conocimiento, capital intelectual, grupos de interés (stakeholders) y sustentabilidad de las organizaciones, entre otros.

El Proceso Administrativo (PA) utiliza las funciones de planear, organizar, liderar y controlar de forma sistemática, integral, compleja e interdependiente, para lograr los objetivos planeados. En este sentido, los autores Stoner, Freeman y Gilbert (1996), aclaran que, en la práctica, el proceso administrativo no incluye cuatro conjuntos aislados o poco conexos de actividades, sino un grupo de funciones interrelacionadas. Las funciones administrativas consideradas como un todo integrado conforman el proceso administrativo; cuando la planeación, la 
organización, la dirección y el control se consideran de manera aislada son denominadas funciones administrativas (Chiavenato, 2013). El enfoque del PA es totalizador, porque las partes del mismo son elementos interactuantes; las causas y efectos repercuten entre ellos (Hernández y Palafox, 2012).

En ese sentido, se puede entender, que el proceso administrativo es un conjunto de operaciones de gestión administrativas cíclicas, sistémicas, holísticas, interdependientes y flexibles. Deben lógicamente, según las características internas y externas de la organización, adaptarse a las necesidades propias de cada organización social en donde se aplican. De esta manera, se cumple en este proceso la característica de "morfogénesis", propias de los sistemas abiertos (organizaciones), de modificar su estructura básica para responder mejor a los estímulos internos y externos.

\subsection{Clasificación de los elementos del proceso administrativo}

Muchos autores (clásicos y neoclásicos), han realizado aportaciones sobre los elementos que conforman el proceso administrativo, desde Fayol, a principios del Siglo XX, hasta Peter Drucker a mediados de los cincuenta, con la teoría neoclásica de la administración. A continuación, se describe en forma breve, según Chiavenato (2013), las cuatro actividades gerenciales principales del proceso administrativo.

a. La planeación: Es la función donde se definen los objetivos por alcanzar y qué se debe hacer para lograrlos.

b. La organización: En esta función se diseña el trabajo, se asignan los recursos y se coordinan las actividades.

c. La dirección: Está directamente relacionada con designar a las personas, dirigir sus esfuerzos hacia un compromiso común, motivarlos y comunicarse con ellos.

d. El control: Esta función tiene como objetivo asegurar que los resultados de las funciones anteriores se ajusten lo más posible a los objetivos establecidos previamente.

De las generalizaciones anteriores, se puede concluir que, el proceso administrativo, es sin duda la metodología que se debe utilizar para gestionar de formar efectiva, coherente, flexible e integral cualquier organización. Es necesario que las organizaciones, utilicen el proceso administrativo como una herramienta para integrar las diferentes actividades que hacen posible el logro de la estrategia empresarial; es decir, debe ser planificada, organizada, dirigida y controlada la acción empresarial - administrada - que pondrá en práctica la estrategia seleccionada.

\section{Sostenibilidad empresarial}

La década de los setenta, es principalmente transcendental por el interés científico respecto al desarrollo económico equilibrado y respetuoso con el medio ambiente. El informe Meadows de 1972, y, especialmente, la crisis del petróleo en 1973, hacen visible los serios problemas a los que ha llevado el modelo de crecimiento económico ilimitado y, sirven para llamar la atención en el desinterés que el discurso económico dominante había tenido hasta entonces sobre las cuestiones ambientales.

Sobre este aspecto, Sachs (2015) expresa: "La gigantesca economía mundial está provocando una gigantesca crisis ambiental, capaz de amenazar la vida y el bienestar de miles de millones de personas en el planeta" (p.19). Debe señalarse que, no es hasta finales de los ochenta, cuando se activa el debate sobre la sostenibilidad, la cual pasa a ser el centro de atención, convirtiéndose desde entonces en uno de los principales temas de investigación, por parte de la comunidad científica, y de interés público y social a nivel mundial.

El concepto de sostenibilidad surge por vía negativa, como resultado de los estudios de la situación del mundo, que puede describirse como una emergencia planetaria. En este sentido, Bybee (1991), comenta que la sostenibilidad constituye la idea central 
unificadora más necesaria en este momento de la historia de la humanidad. La cual, se apoya en el estudio de los problemas, en el análisis de sus causas y en la adopción de medidas correctoras. Existen unas condiciones insostenibles: Pobreza, desequilibrio ecológico, transculturación, entre otras, que amenazan gravemente el desarrollo de la humanidad $\mathrm{y}$, que deben ser atendidas con urgencia.

Sánchez y Pulido (2008), comentan que la sostenibilidad implica un cambio del paradigma en el aprovechamiento de los recursos, reconociendo que existen límites a la disponibilidad de los mismos y, por lo tanto, deben tomarse medidas para reducir el consumo material que eleva los costes ambientales, culturales y sociales. En este mismo orden de ideas, los autores Campo-Ternera, et al. (2018), señalan que el desarrollo sostenible, es un tema que obliga tener en cuenta aspectos económicos, sociales y medioambientales, frente a los cuales se presenta la necesidad de generar una cultura de empresarios enfocados en un crecimiento económico sostenible, que incluya una mayor cohesión y justicia social.

De las generalizaciones expuestas, se puede resumir, que el proceso de sostenibilidad contempla un sentido económico - social ambiental, destacando siempre la situación ecológica y social causada por la producción económica, definiendo claramente cuáles deben ser las condiciones adecuadas para cada una de las dimensiones. Se insiste en la necesidad de una gestión sostenible y uso eficiente de los recursos naturales. En efecto, los autores Guillén, et al. (2020), afirman que: "El camino que ha venido recorriendo el desarrollo sostenible, se sustenta en el mejor manejo de los recursos naturales, no en su explotación" (p.297).

El concepto de sostenibilidad tiene ámbitos de aplicación muy diferentes. Desde una perspectiva sectorial, es posible hablar de turismo sostenible, agricultura y empresas sostenibles. En la agenda 2030: Objetivos de Desarrollo Sostenible (ODS), su objetivo 9 está relacionado con: Construir infraestructura resiliente, promover la industrialización inclusiva y sostenible y fomentar la innovación (ONU, 2020).

En ese sentido, el desarrollo sostenible depende de una buena infraestructura pública y privada. En el caso de la industria hotelera, el sector puede influir en las políticas públicas de modernización de la infraestructura, haciéndolas más sostenibles, innovadoras y eficientes en materia de recursos y avanzando hacia un crecimiento bajo en carbono, atrayendo así a los turistas así como otras fuentes de inversión extranjera. Por ejemplo, ecodiseño; nuevos equipos y tecnologías en recursos energéticos e hídricos; energías renovables: Equipos y materiales para reciclaje de residuos.

Para las empresas, mantener la sostenibilidad en un contexto de crecimiento económico y desarrollo, se ha vuelto el reto más importante al que se están enfrentando en la actualidad, puesto que implica la aplicación de aspectos normativos y éticos, que, en algunos casos, contradicen los intereses de las empresas. Según Epstein (2009), las compañías líderes cada vez más reconocen la importancia en administrar y controlar el desempeño corporativo de carácter social y ambiental. Numerosas compañías han dado pasos importantes hacia el mejoramiento de la sostenibilidad de su desempeño y a reducir sus impactos sociales y ambientales negativos.

Algunos autores exponen el significado de sostenibilidad empresarial. Gil y Barcellos (2011), explican que una empresa es sostenible cuando es capaz de generar valor económico, ambiental y social a mediano y largo plazo, contribuyendo de esa forma al aumento del bienestar y al auténtico progreso de las generaciones presentes y futuras. Uribe-Macias, Vargas-Moreno y MerchánParedes (2018), explica que la sostenibilidad empresarial muchas veces es interpretada por los empresarios o gerentes de proyectos como un camino de aprendizaje, con expresión muy difusa que no es suficiente para establecer claramente programas a corto plazo que incluyan las partes interesadas y que den una estructura definida al tema.

Por otra parte, Epstein (2009), presenta 
una definición amplia de sostenibilidad empresarial, centrándose en nueve criterios que usualmente se incluyen en las discusiones, análisis, medición y reportes sobre la sostenibilidad social, ambiental y financiera de la empresa. Estos criterios que conforman la definición son los siguientes: Ética, gobierno, transparencia, relaciones de negocios, retorno financiero, participación de la comunidad, valor de los productos y servicios, prácticas de empleo y protección del ambiente. Se puede interpretar, que los criterios que expone este autor son los principios que orientan la acción empresarial para el logro equilibrado de un desarrollo sostenible de la organización.

Se puede resumir a continuación, que la sostenibilidad empresarial debe ser entendida como una acción ética y básica (planificada, organizada, dirigida y controlada) para estimular la sensibilidad de la organización hacia los problemas sociales y ambientales, la cual da oportunidad a reducir los impactos negativos de la empresa hacia la sociedad, y a su vez, aumentar la rentabilidad corporativa a largo plazo.

\section{El sector hotelero de la parroquia Crucita, provincia de Manabí-Ecuador}

Crucita, es una parroquia rural del cantón Portoviejo. Por sus características geográficas, Crucita posee un enorme potencial de desarrollo turístico, cuenta con trece kilómetros de playa y con actividades complementarias para una oferta turística diversificada. Sin embargo, el desarrollo de esta actividad se ha llevado a cabo en un ambiente de escasa planificación y ha sido más producto de iniciativas aisladas, en su mayoría proveniente del sector privado. Crucita se encuentra a $30 \mathrm{~km}$. de la ciudad de Portoviejo, siendo esta última la capital de la provincia de Manabí. En el 2010 esta comunidad rural contaba con una población de 14.050 habitantes (Instituto Nacional de Estadística y Censos [INEC], 2010).

En los últimos años ha aumentado el interés de nacionales y extranjeros en la construcción de un número de casas de hospedaje y hoteles en la parroquia Crucita. El alquiler de las habitaciones ha permanecido bastante bajo, promediando un costo de $\$ 20,00$ por habitación y $\$ 10,00$ por persona, y en hoteles más cómodos hasta $\$ 40,00$ por habitación y $\$ 20,00$ por persona (Huiza, Cruz y Martínez, 2010; Coral, 2015). El sector hotelero de Crucita está compuesto por un total de 29 hoteles, según el censo realizado por la Asociación de Empresarios Hoteleros de Crucita en 2019 (Márquez, et al., 2020).

En el sector hotelero de Crucita es común observar los siguientes servicios: Bar, lavandería, restaurante, piscina, servicios de habitación, baño con agua caliente, teléfono, caja de seguridad, internet inalámbrico, aire acondicionado y televisión (Trejo, 2013). La parroquia Crucita, no cuenta con un hotel que brinde de manera integral todos los servicios antes mencionados, sino que están dispersos entre la infraestructura existente, y que además no cumplen con los estándares de calidad necesario para satisfacer las expectativas de los clientes.

En conversaciones con los miembros de la Asociación de hoteleros de la Parroquia, éstos detallan un conjunto de aspectos que limitan el aumento de sostenibilidad en lo social (mayor empleo), económico (mayor rentabilidad) y ambiental (deterioro de la playa como principal recurso para ofertar). Esta situación le resta oportunidades de desarrollo y competitividad al sector hotelero con relación a otros destinos turísticos, como son: Puerto Cayo, Puerto López, Jama y Canoa, destinos de sol y playa importantes de la provincia de Manabí.

En este sentido, es necesario que la administración hotelera de Crucita defina estrategias para mejorar su nivel de competitividad, lo cual mejorará los impactos corporativos sociales, económicos y ambientales, así como acciones responsables del sector hacia la comunidad. De esta manera, lo explica Epstein (2009), la evaluación de los impactos sociales, económico y ambientales de las acciones organizacionales es necesaria para tomar decisiones efectivas de carácter 
operacional y de inversión del capital, que impacten de manera positiva los objetivos organizacionales y satisfagan los objetivos de los múltiples stakeholders. En muchos casos, la reducción de esos impactos incrementa la rentabilidad corporativa en el largo plazo mediante rendimientos de producción más altos y calidad mejorada del producto.

\section{Metodología}

Dentro del contexto de estudio, la presente investigación tiene como finalidad determinar el nivel de relación entre el proceso administrativo y la sostenibilidad empresarial del sector hotelero de la parroquia Crucita del cantón Portoviejo en Manabí-Ecuador. Esta propuesta posee una metodología estructurada por etapas, elaborada a partir de un proceso de revisión bibliográfica, elaboración matricial con las principales propuestas de indicadores identificados, utilización de la técnica de expertos (o método Delphi) y tabla de indicadores para medir cada una de las variables de estudio y presentación de los resultados. En la Figura I, se presenta el proceso metodológico utilizado para la investigación.

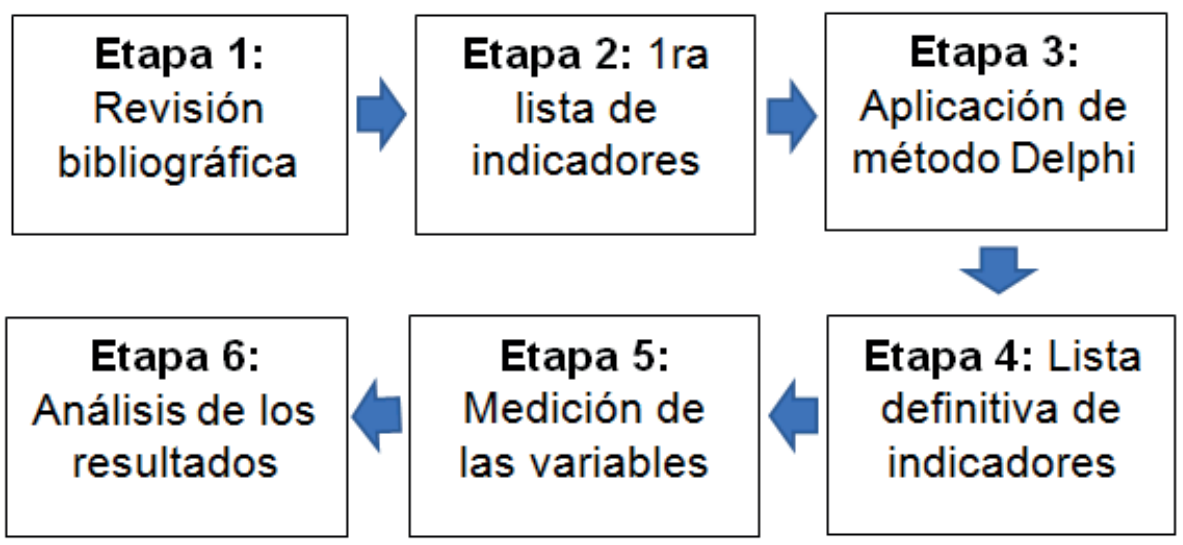

Fuente: Elaboración propia, 2020.

Figura l: Proceso metodológico para el desarrollo de la investigación

Los hoteles seleccionados para el estudio son: Ocean Blue, Marlin, Java, Washington, Hipocampo, Casa Grande y Gran Oasis. El criterio para elegir este grupo de hoteles, del total de 29 hoteles en la parroquia Crucita, fue de incluir en el estudio los hoteles con veinte o más habitaciones.

En la Etapa 1, se efectúa la revisión bibliográfica o investigación documental, la cual se realiza a partir de datos secundarios, éstos son fuentes de información necesaria para la elaboración de la primera lista de indicadores. La Etapa 2, contempla elaborar una lista de indicadores que serán validados por los expertos a partir de la aplicación del método Delphi.

Con relación a la Etapa 3, en ella se aplica la técnica de expertos (o método 
Delphi) a la 1ra lista de indicadores que han resultado de la revisión bibliográfica. Esta técnica, se utiliza cuando el objeto de estudio no está lo suficientemente estructurado (Goicoechea, Hansen y Duckstein, 1982). En esta etapa, primero se calcula el coeficiente de competencias, el cual se determina de acuerdo con la opinión del experto sobre su nivel de conocimiento con relación al problema que se requiere resolver (o temática de estudio), y con las fuentes que le permiten comprobar su valoración. El coeficiente de competencia se calcula de la siguiente forma:

$\mathrm{K}=(\mathrm{Kc}+\mathrm{Ka}) / 2$,

Donde: Kc, es el coeficiente de conocimiento o información que tiene el experto respecto al problema o tema, calculado sobre la valoración del propio experto; y $\mathrm{Ka}$, es el coeficiente de argumentación o fundamentación de los criterios del experto.

Luego de aplicar el coeficiente de competencias $\mathrm{y}$, tener seleccionado a los expertos que validarán los indicadores identificados en la Etapa 2, se establece un diálogo anónimo e individual entre los expertos seleccionados para la validación, y se le aplica un cuestionario relacionado a la selección de indicadores para evaluar cada una de las variables de estudio. Al final del proceso, se obtiene la lista definitiva de indicadores mediante la aplicación del método de consenso, el cual indica cuales son los seleccionados para medir las variables a partir de la puntuación de votos otorgada por cada uno de los expertos, que establece como criterio de decisión un valor porcentual igual o mayor al $80 \%$ de aceptación.

Acerca de la Etapa 4, aquí se elabora la lista definitiva de indicadores que servirán para medir cada variable objeto de estudio. La Etapa 5, corresponde al trabajo de campo, el cual consiste en visitar en la parroquia Crucita a cada uno de los siete hoteles seleccionados para el estudio, con el fin de recopilar la información según los indicadores validados por los expertos. En la Etapa 6, se procesa la información con el programa estadístico informático SPSS versión 25, y se determina la correlación entre las variables a partir del coeficiente de correlación de Pearson. Finalmente, se presentan los resultados de forma didáctica en un diagrama radial, histograma, gráfico de líneas y diagrama de dispersión.

\section{Resultados}

\subsection{Indicadores del proceso administrativo}

El primer resultado obtenido, según el proceso metodológico, es la lista de indicadores para cada una de las variables objeto de estudio. Seguidamente, en el Cuadro 1 , se muestra la lista de diecinueve indicadores de la variable "proceso administrativo", la cual se logra a partir de la revisión bibliográfica y su validación con el método Delphi.

\section{Cuadro 1 \\ Indicadores del proceso administrativo}

\begin{tabular}{l} 
Indicadores del proceso administrativo \\
\hline 1. Aplicación de la planificación estratégica a nivel general. \\
2. Aplicación de planes tácticos por departamento en el hotel. \\
3. Asignación de presupuesto por actividad a nivel de departamento. \\
4. Aplicación de planes operacionales (planificación de actividades específicas). \\
5. Presupuesto por actividad (asignación de presupuesto para la ejecución de actividades específicas). \\
6. Estructura organizativa (organigrama general del hotel). \\
\hline
\end{tabular}




\section{Cont... Cuadro 1}

7. Estructura de departamentos y cargos (organigrama por departamento y cargos).

8. Descripción de cargos (enumeración de tareas o atribuciones que conforman un cargo).

9. Nivel de definición de procedimientos administrativos en el hotel.

10. Nivel de aplicación de métodos y procesos de trabajo en el área operacional.

11. Aplicación de políticas y directrices para la gestión del personal.

12. Aplicación de recursos para materializar la estrategia empresarial y lograr el liderazgo del hotel en el sector.

13. Nivel de supervisión del personal.

14. Nivel de motivación del personal.

15. Nivel de reconocimiento por parte de la dirección a los trabajadores.

16. Nivel de capacitación del personal por parte de la dirección del hotel.

17. Controles y evaluación del desempeño global del hotel.

18. Controles y evaluación del desempeño por departamento.

19. Controles y evaluación del desempeño individual.

Fuente: Elaboración propia, 2020 a partir de Stoner, et al. (1996); Hernández y Palafox (2012); y, Chiavenato (2013).

\subsection{Comparación del proceso administrativo} Para establecer las comparaciones de diecinueve indicadores (ver Gráfico l). los procesos administrativos en cada uno de los hoteles, se procede a mostrar mediante diagramas radiales la evaluación de los

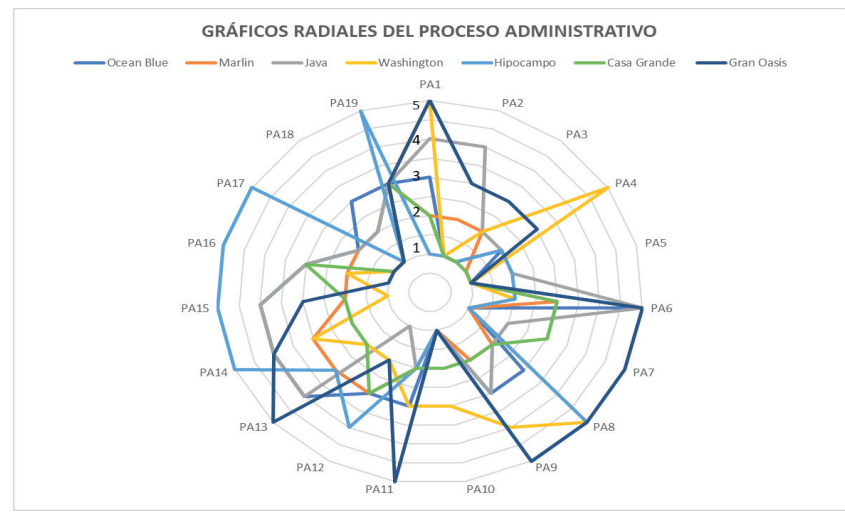

Fuente: Elaboración propia, 2020.

\section{Gráfico I: Gráficos radiales del proceso administrativo}


Se aprecia en el Gráfico I, los radiales de los hoteles con respecto al proceso administrativo, recalcando que los hoteles que más resaltan por sus indicadores, en la mayoría "muy altos", son el hotel Gran Oasis, hotel Hipocampo y el hotel Washington, aunque no en la totalidad de sus indicadores. Por otra parte, el hotel Marlin y Casa Grande, alcanzan los menores puntajes en sus indicadores.

\subsection{Indicadores de sostenibilidad empresarial}

El proceso metodológico, igualmente permitió definir los indicadores para la variable "sostenibilidad empresarial". A continuación, en el Cuadro 2, se muestra la lista de diecinueve indicadores, la cual se logra a partir de la revisión bibliográfica y su validación con el método Delphi.

\section{Cuadro 2 \\ Indicadores de sostenibilidad empresarial}

\section{Indicadores de sostenibilidad empresarial}

1. Aplicación de códigos de conductas responsables en el hotel.

2. Realización de talleres y capacitaciones sobre comportamiento ético y responsable en hotel.

3. Aplicación de la Norma internacional de Responsabilidad Social SA 8000 en el hotel.

4. Nivel de información y dominio por parte de los empleados de la misión, estrategia, políticas, prácticas y procedimientos responsables del hotel.

5. Realización de reuniones con los accionistas, trabajadores, comunidad y gobierno.

6. Nivel de información a los inversionistas, prestamistas y empleados sobre el desempeño financiero pasado, presente y futuro del hotel.

7. Nivel de conocimiento sobre el impacto económico, social y ambiental del hotel, y que informa a los accionistas, trabajadores, comunidad y gobierno.

8. Nivel de relación entre la empresa hotelera y los proveedores.

9. Aplicación de convenios entre el hotel y los proveedores para estimular la calidad y el desempeño social, ético y ambiental.

10. Retorno sobre la inversión y la protección de los activos de los inversionistas.

11. Participación del hotel en proyectos de la comunidad relacionados con la educación, salud, ambiente, infraestructura y cultura.

12. Realización de estudios para conocer el nivel de satisfacción del cliente hacia los productos y servicios ofrecidos por el hotel.

13. Aplicación de programas para incrementar los niveles de satisfacción de los empleados con relación a las prácticas razonables y seguras de trabajo, salarios y beneficios competitivos, entornos seguros y amigables con la familia.

14. Desarrollo de productos, procesos, servicios y actividades realizadas por el hotel para conservar el ambiente. 
Márquez Ortiz, Luis Enrique; Viteri Mero, Morelia Jessenia; Useche Castro, Lelly María y Cuétara Sánchez, Leonardo Manuel

Proceso administrativo y sostenibilidad empresarial del sector hotelero de la parroquia

Crucita, Manabi-Ecuador

\section{Cont... Cuadro 2}

15. Cumplimiento de las regulaciones locales, nacionales e internacionales, relacionadas con emisiones y desechos sólidos y líquidos.

16. Ingreso proveniente de productos reciclados

17. Percepción de los clientes sobre el desempeño ético del hotel

18. Certificaciones ambientales y de calidad del hotel

19. Trabajos locales generados

Fuente: Elaboración propia, 2020, a partir de Epstein (2009); Urruticoechea y Vernazza (2019); y, Martínez, et al. (2019).

\subsection{Comparación de la sostenibilidad empresarial}

La comparación de la sostenibilidad empresarial en cada uno de los hoteles evaluados se realiza mediante diagramas radiales (ver Gráfico II), con los diecinueve indicadores seleccionados para el estudio.

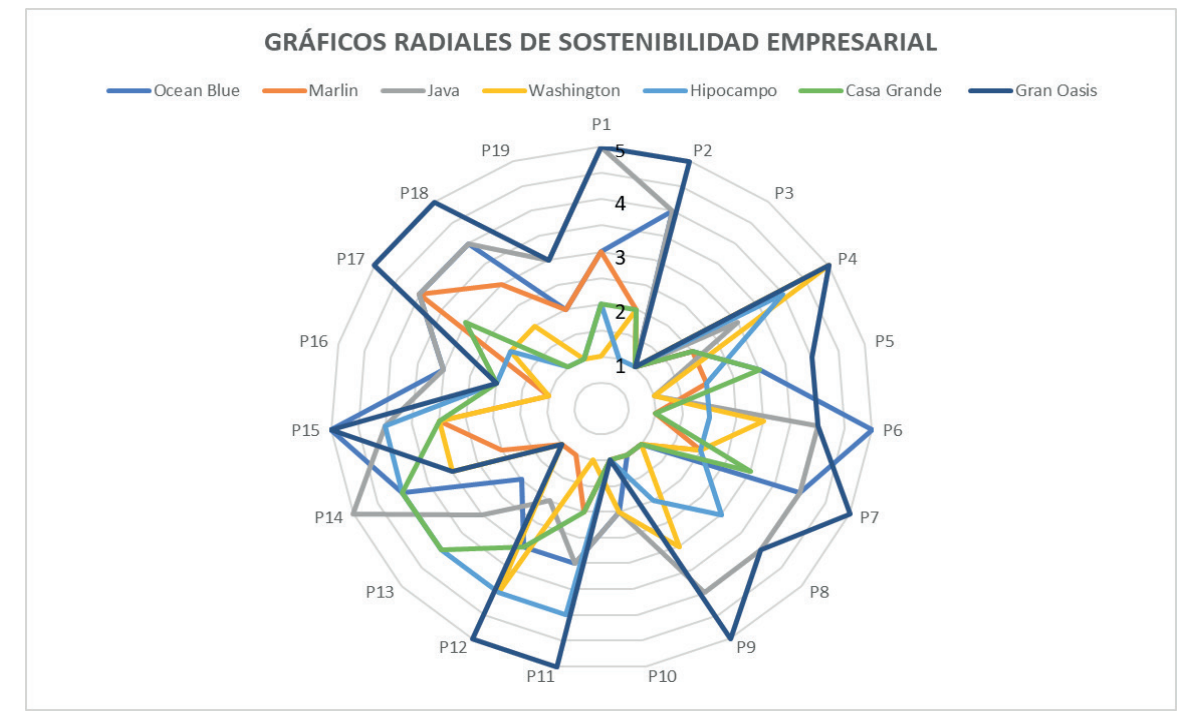

Fuente: Elaboración propia, 2020.

Gráfico II: Gráficos radiales de sostenibilidad empresarial 
Observando el Gráfico II, del comportamiento de los hoteles con respecto a la sostenibilidad empresarial, se aprecia que todos los hoteles tienen resultados diferentes, se destaca el hotel Gran Oasis nuevamente, como ocurrió con el proceso administrativo, con la mayoría de sus indicadores en el nivel "muy altos", mientras que el hotel Washington y Marlin con indicadores bajos, a pesar de que este último, tuvo mejores resultados en el proceso administrativo. Por otra parte, el hotel Hipocampo que tiene varios indicadores del proceso administrativo "muy alto", en esta oportunidad, no presenta ninguno, sin embargo, en líneas generales tiene indicadores "altos".

\subsection{Comparación integral del proceso administrativo y la sostenibilidad empresarial}

Con la idea de mostrar el comportamiento de la variable "proceso administrativo" en cada hotel con relación al grupo, se presenta en el Gráfico III un histograma integral, donde se puede observar la magnitud de los indicadores en cada escala según el hotel evaluado.

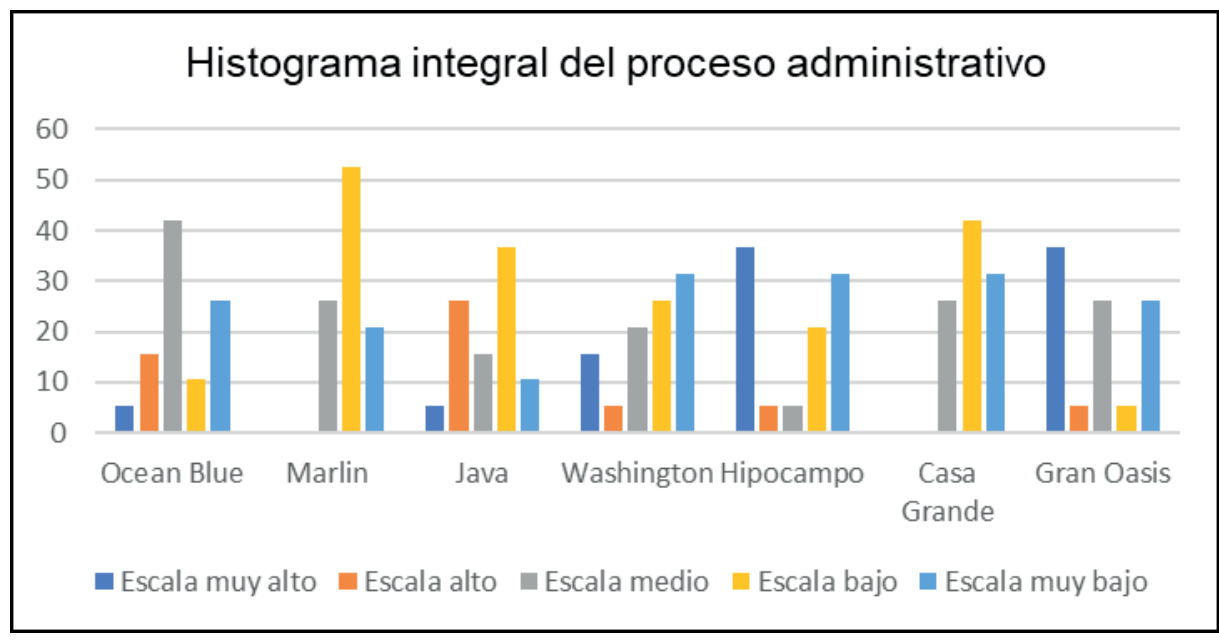

Fuente: Elaboración propia, 2020.

\section{Gráfico III: Histograma integral del proceso administrativo}

El histograma integral del proceso administrativo muestra que, los hoteles con el mayor número de indicadores activos en el proceso administrativo en la escala "muy alto" son el Hipocampo y Gran Oasis, ambos obtuvieron $36,8 \%$ en esta escala. Igualmente, para la escala "alto", ambos hoteles lograron un 5,2\% de indicadores presentes en este nivel; asimismo, el hotel Java alcanzó un $26,3 \%$ en la escala "alto". A diferencia de los hoteles
Marlin y Casa Grande, los cuales no muestran ningún indicador del proceso administrativo en la escala "muy alto" y "alto".

En el Gráfico IV, se muestra a continuación el histograma integral de la variable "sostenibilidad empresarial" para cada uno de los siete hoteles evaluados, donde se puede observar el nivel en porcentaje de cada escala según los indicadores contenidos en ella. 


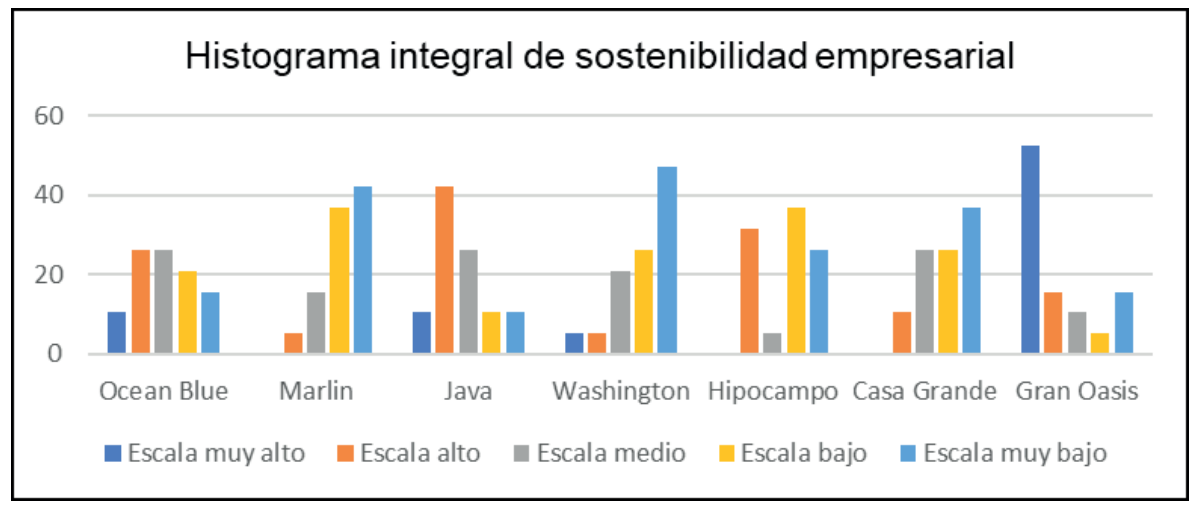

Fuente: Elaboración propia, 2020.

\section{Gráfico IV: Histograma integral de la sostenibilidad empresarial}

Según el histograma integral de sostenibilidad empresarial, el mejor comportamiento a nivel de sostenibilidad lo obtuvo el hotel Gran Oasis, con un 52,6\% de indicadores presentes en la escala de "muy alto" y $15,7 \%$ en la escala "alto". El hotel Java, es el segundo hotel con mejor rendimiento sostenible, el cual logró $10,5 \%$ de indicadores en la escala "muy alto" y $42,1 \%$ en la escala "alto". Mientras que, los hoteles con niveles de sostenibilidad deficientes son el Marlin, que consiguió un 5,2\% de indicadores en la escala "alto" y el hotel Casa Grande, quien obtuvo $10,5 \%$ en la misma escala.

\subsection{Relación entre el proceso administrativo y la sostenibilidad empresarial}

Con la finalidad de poder establecer una comparación o determinar la relación entre el "proceso administrativo" y la "sostenibilidad empresarial", en el Gráfico V, se aprecia los totales de los indicadores obtenidos por hotel en los procesos administrativos y la sostenibilidad empresarial, y se observa cierto patrón de comportamiento entre ambas variables, es decir, hoteles con puntaje alto en proceso administrativo, tiene puntaje alto en sostenibilidad empresarial y viceversa, lo cual pudiera indicar que si hay una relación entre ambas variables. Para ello, lo más conveniente es hacer uso de un estadístico de prueba para corroborar tal relación. 


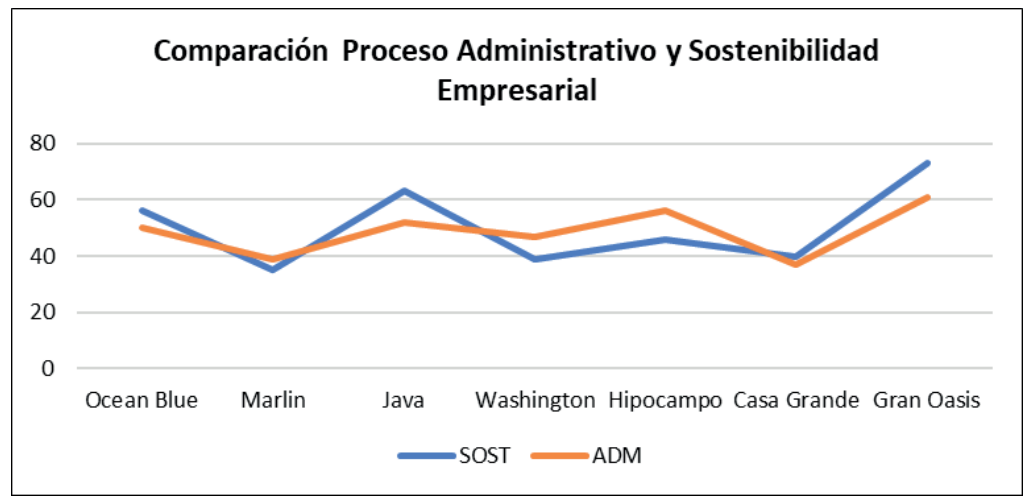

Fuente: Elaboración propia, 2020.

\section{Gráfico V: Gráfico de líneas de los totales de los indicadores de proceso administrativo y sostenibilidad empresarial}

Ahora bien, con la finalidad de comparar si los datos tienen un comportamiento normal, para llevar a cabo el coeficiente de correlación de Pearson, se prueba mediante el estadístico de Shapiro-Wilks, quien da con un p-valor mayor a 0,05 en ambas variables, para la sostenibilidad empresarial de 0,489 y para el proceso administrativo de 0,824 , indicando que, aunque son pocos hoteles en el estudio siguen una distribución normal y puede hacerse uso del estadístico de Pearson para establecer la relación entre ambas variables.

En efecto, la correlación entre ambas variables es de 0,797 indicando que, si hay una relación directamente proporcional alta entre ambas variables, es decir, que a medida que hay altos puntajes en los procesos administrativos, hay altos puntajes en la sostenibilidad empresarial. En consecuencia, lo anteriormente descrito se puede corroborar en el Gráfico VI, en donde se aprecia que el hotel Hipocampo presenta mayor sostenibilidad con menor valor de procesos administrativos. Este gráfico también permite, de cierta manera, ordenar los hoteles según cualquiera de los dos indicadores, por ejemplo, con respecto a la sostenibilidad los hoteles se pueden ordenar desde el "más bajo" al "más alto"; Casa Grande, Marlin, Whashington, Ocean Blue, Java, Hipocampo y Gran Oasis.

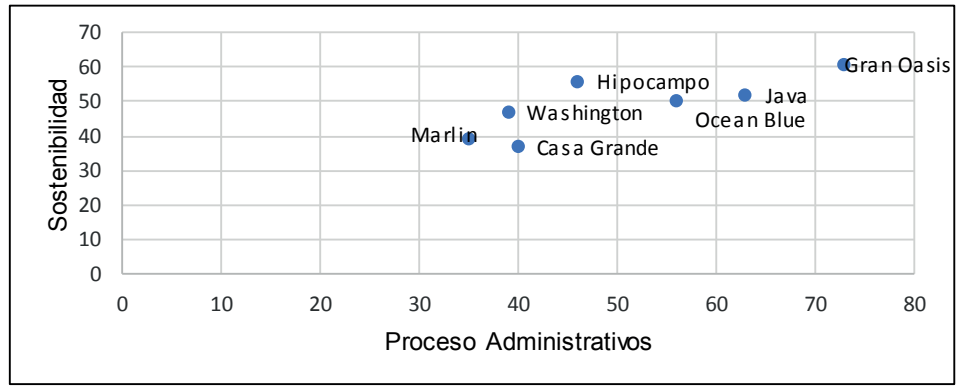

Fuente: Elaboración propia, 2020.

Gráfico VI: Gráfico de dispersión del proceso administrativo y sostenibilidad empresarial 


\section{Discusión}

En los hoteles seleccionados para el estudio se observa que el proceso administrativo no es considerado de forma global para alcanzar los objetivos de la organización; sin embargo, las funciones del proceso administrativo son utilizadas de forma aislada, en niveles y áreas específicas. Los hoteles que mejor aplican esta herramienta para integrar las diferentes actividades empresariales obtienen mejores resultados. Lógicamente, esta realidad apoya los aspectos teóricos de los autores Fayol (1987); Stoner, et al. (1996); Chiavenato (2006); Hernández y Palafox (2012), los cuales coinciden con el uso necesario del proceso administrativo para el logro de los objetivos de la organización.

El promedio de aplicación del proceso administrativo en los hoteles evaluados de la parroquia Crucita es de un $14,2 \%$ en la escala de "muy alto" y de un $8,2 \%$ en la escala "alto". Siendo los hoteles Hipocampo y Gran Oasis, los que más aplican el proceso administrativo como herramienta para alcanzar sus objetivos. Entre los aspectos relacionados a la gestión del proceso administrativo para la puesta en práctica de la sostenibilidad; se destaca que, cuando mayormente se ejecutan las funciones administrativas (planificación, organización, dirección y control) mayores posibilidades tiene el hotel para atender las necesidades de la empresa y del ambiente que la rodea.

En teoría, el sector hotelero de Crucita busca aumentar su rentabilidad económica, pero debe lograr un modelo de sostenibilidad más integral; planificando, organizando, dirigiendo y controlando una estrategia económica, social, ambiental competitiva. Al respecto, Elkington (1999), reconocido mundialmente como el "padre de la sostenibilidad", lo expresa de esta forma, el equilibrio entre los beneficios económicos, la responsabilidad social y la protección ambiental, puede conducir a ventajas competitivas. Evidentemente, lo que plantea aplica perfectamente al sector hotelero, puesto que los hoteles dependen de un entorno natural y social conservado para generar rentabilidad económica.

Los hoteles de Crucita, obligatoriamente deben realizar operaciones para preservar los entornos naturales y sociales que los rodea. Ellos también son responsables de colocar en práctica acciones para conocer los impactos sociales, económicos y ambientales generados interna así como externamente. Los autores Bybee (1991); BID (2007); Sánchez y Pulido (2008); Epstein (2009); Gil y Barcellos (2011); Sachs (2015); Uribe, et al. (2018); ONU (2020); exponen la urgencia de la sostenibilidad como estrategia para mejorar las condiciones de la sociedad. La Red de Soluciones para el Desarrollo Sostenible (SDSN) Australia, Nueva Zelanda y Pacífico, explica que La Agenda 2030 reconoce que abordar la sostenibilidad es responsabilidad de todos. Por tanto, hace un llamado explícito a las entidades públicas, privadas, a la sociedad civil y al sector académico, entre otros, para que aporten en su consolidación (SanabriaSuárez, et al., 2020).

Con relación al nivel de sostenibilidad empresarial del sector hotelero de la parroquia Crucita, el promedio logrado por los hoteles evaluados es de $11,2 \%$ en la escala "muy alto" y un $15,7 \%$ en la escala "alto". El hotel que obtiene mejor comportamiento en correspondencia a la sostenibilidad, es el hotel Gran Oasis. Asimismo, el coeficiente de correlación de Pearson permitió demostrar que si hay relación alta entre la variable "Proceso administrativo" y "Sostenibilidad empresarial". Es decir, en el caso de los hoteles evaluados, la sostenibilidad empresarial si es influenciada por el nivel de aplicación del proceso administrativo.

Debe señalarse, que la sostenibilidad debe ser un componente integral de la estrategia corporativa en los hoteles de la parroquia Crucita. El administrador de cada hotel tiene la obligación legal, ética y económica de comprometerse con la sostenibilidad, y construir capacidad organizacional para colocarla en práctica a partir del proceso administrativo, esto los ayudará, como lo expone Elkington (1999), a lograr ventajas competitivas. 


\section{Conclusiones}

A partir de los resultados del estudio efectuado, y cumpliendo con todos los parámetros establecidos en los objetivos, se puede establecer las siguientes conclusiones: en función de los hallazgos y el análisis de los datos, se pudo determinar que la mayoría de los hoteles evaluados no aplican el proceso administrativo de forma global e integral.

Asimismo, se pudo evidenciar que la sostenibilidad en el proceso administrativo de los hoteles no es considerada una estrategia; en este caso, los niveles positivos de sostenibilidad logrados por algunos de los hoteles evaluados, es producto del cumplimiento de los aspectos legales.

De igual manera, se encontró que los hoteles con mejor rendimiento sostenible son los más competitivos a nivel general; es decir, se da una relación directamente proporcional alta entre las variables "proceso administrativo" y "sostenibilidad empresarial". Sucede que, a medida que hay altos puntajes en los procesos administrativos, también se evidencian niveles altos en la sostenibilidad empresarial.

\section{Referencias bibliográficas}

Alcívar, I. I. (2018). Turismo en la economía de Ecuador. Entorno Turístico. https:// www.entornoturistico.com/turismoen-la-economia-de-ecuador/

Banco Interamericano de Desarrollo - BID (2007). Diseño del Plan Estratégico de Desarrollo de Turismo Sostenible para Ecuador "PLANDETUR 2020". Informe final. MINTUR, BID. https://www.turismo.gob.ec/wpcontent/uploads/downloads/2013/02/ PLANDETUR-2020.pdf

Bybee, R. W. (1991). Planet Earth in crisis: how should science educators respond? The American Biology Teacher, 53(3), 146153. https://doi.org/10.2307/4449248
Campo-Ternera, L., Amar-Sepúlveda, P., Olivero, E., y Huguett, S. (2018). Emprendimiento e innovación como motor del desarrollo sostenible: Estudio bibliométrico (2006- 2016). Revista de Ciencias Sociales (Ve), XXIV(4), 26-37.

Chiavenato, I. (2001). Administración: Proceso administrativo. McGrawHill/ Interamericana de Colombia, S.A.

Chiavenato, I. (2006). Introducción a la teoría general de la administración 7ma. Edición. McGraw-Hill/ Interamericana, S.A.

Chiavenato, I. (2013). Introducción a la teoría general de la administración. 8va, Edición. McGraw-Hill/ Interamericana, S.A.

Coral, S. (2015). Plan de Desarrollo y Ordenamiento Territorial de la Parroquia Crucita: Diagnóstico. GAD de Crucita. https://bit.ly/3uAkrqn

Corporación Financiera Nacional - CFN (2017). Ficha sectorial: Sector turístico - nivel nacional. CFN. https://www.cfn.fin.ec/wp-content/ uploads/2017/10/Ficha-SectorialTurismo.pdf

Elkington, J. (1999). Cannibals with forks: Triple bottom line of 21 st century business. John Wiley \& Son Ltd.

Epstein, M. J. (2009). Sostenibilidad empresarial: Administración $y$ medición de los impactos sociales, ambientales y económicos . ECOE Ediciones.

Fayol, J. H. (1987). Administración industrial y general: Principios de la administración cientifica. El Ateneo Editorial.

Gil, A. M., y Barcellos, L. (2011). Los desafíos para la sostenibilidad empresarial en el siglo XXI. Revista Galega de 
Márquez Ortiz, Luis Enrique; Viteri Mero, Morelia Jessenia; Useche Castro, Lelly María y

Cuétara Sánchez, Leonardo Manuel

Proceso administrativo y sostenibilidad empresarial del sector hotelero de la parroquia

Crucita, Manabi-Ecuador

Economía, 20(2), 1-22.

Goicoechea, A., Hansen, D. R., y Duckstein, L. (1982). Multiobjetive decision analysis with engineering and business applications. John Wiley and Sons.

Guillén, J., Calle, J., Gavidia, A. M., y Vélez, A. G. (2020). Desarrollo sostenible: Desde la mirada de preservación del medio ambiente colombiano. Revista de Ciencias Sociales (Ve), XXVI(4), 293-307. https://doi.org/10.31876/rcs. v26i4.34664

Gutiérrez, J. (2009). Reporte de sostenibilidad 2009. ECOPETROL.

Hernández, S., y Palafox, G. (2012). Administración: Teoría, proceso, áreas funcionales y estrategias para la competitividad. McGraw-Hill.

Huiza, F., Cruz, K., y Martínez, V. (2010). Plan estratégico de desarrollo turístico de Crucita 2010-2014. Consejo Provincial de Portoviejo. https://www.researchgate.net/profile/ Kerly-Cruz/publication/311452011 PLAN ESTRATEGICO DE DESARROLLO TURISTICO D E C R U C I T A $20010 /$ links/5846ede608ae61f75ddfdf62/ PLAN-ESTRATE G I C O-DEDESARROLLO-TURISTICO-DECRUCITA-2010.pdf

Instituto Nacional de Estadística y Censos INEC (2010). Base de Datos-Censo de Población y Vivienda 2010. INEC. https://www.ecuadorencifras.gob.ec/ base-de-datos-censo-de-poblacion-yvivienda-2010/

La Hora (7 de septiembre de 2019). Empleo en el sector turístico aumentó $18 \%$ en 2019. La Hora. https://lahora.com. ec/esmeraldas/noticia/1102270997/ empleo-en-el-sector-turisticoaumento-18 -en-2019.

Márquez, L. E., Cuétara, L. M., Bernardo, J. L., y Mera, D. A. (2020). Sistema de indicadores para la evaluación de la sostenibilidad económica del sector hotelero en la parroquia Crucita, Manabí, Ecuador. Revista Espacios, 41(3), 3-11.

Martínez, J. A., Reyes, D. M., Carrillo, L. N., y Rueda, M. J. (2019). Implementación de un método de medición de la sostenibilidad empresarial. NOVUM, Revista de Ciencias Sociales Aplicadas, 2(9), 49-64.

Ministerio de Turismo del Ecuador - MINTUR (7 de febrero de 2019). Turismo aportó con más de 2.300 millones a la economía ecuatoriana en 2018. Ministerio de Turismo. https://www. turismo.gob.ec/turismo-aportocon-mas-de-2-300-millones-a-laeconomia-ecuatoriana-en-2018/

Ministerio de Turismo del Ecuador - MINTUR (2020). Proyecto: "Desarrollo de Destinos y Servicios Turísticos 2018 - 2020”. MINTUR. https:// www.turismo.gob.ec/wp-content/ uploads/2020/02/Anexo-0.-13-11-19 PROY DESTINOS SERVICIOS.pdf

Organización de las Naciones Unidas - ONU (2020). La Agenda para el Desarrollo Sostenible. ONU. https://www. un.org/sustainabledevelopment/es/ development-agenda/.

Organización Mundial del Turismo - OMT (20 de enero de 2019). El turismo internacional sigue adelantando a la economía global. UNWTO. https:// www.unwto.org/es/el-turismomundial-consolida-su-crecimientoen-2019.

Sachs, J. D. (2015). La era del desarrollo sostenible: Nuestro futuro está en juego: incorporemos el desarrollo sostenible a la agenda política mundial. Ediciones Deusto.

Sanabria-Suárez, A. C., Forero, A. M., Rojas, A. L., y Castillo, J. M. (2020). 
Evaluación de las capacidades académicas de las instituciones de educación superior frente a los Objetivos de Desarrollo Sostenible: Una propuesta metodológica. Revista Desarrollo y Sociedad, (86), 133-190. https://doi.org/10.13043/DYS.86.5

Sánchez, M., y Pulido, J. I. (2008). Medida de la sostenibilidad turística: Propuesta de un indice sintético. Editorial Universitaria Ramón Areces.

Serrano, C. (2018). Sector hotelero en crecimiento. Revista Enfoque, (8), 5052.

Stoner, J. A. F., Freeman, R. E., y Gilbert, D. R. (1996). Administración. Pearson Prentice Hall.

Taylor, F. W. (1911). Los principios de la administración cientifica. Harper \& Brothers.
Trejo, D. A. (2013). El desarrollo hotelero y la zona playera: Diseño de una hostería alternativa en la parroquia de Crucita provincia de Manabi (Tesis de pregrado). Instituto Tecnológico "Cordillera", Quito, Ecuador.

Uribe-Macias, M. E., Vargas-Moreno, Ó. A., y Merchán-Paredes, L. (2018). La responsabilidad social empresarial y la sostenibilidad, criterios habilitantes en la gerencia de proyectos. Revista Entramado, 14(1), 52-63.

Urruticoechea, A., y Vernazza, E. (2019). Sostenibilidad empresarial: análisis a través de la metodología biplot. Cuadernos del CIMBAGE, 1(21), 87115.

Vélez, C., y Poveda, E. L. (2017). El sector hotelero portovejense después del terremoto del 16A. Universidad Espíritu Santo. 